\title{
Evaluation of adenosine deaminase activity for diagnosis of tuberculous pleural effusion
}

\author{
Bhoumik SK ${ }^{1}$, Rahman $\mathrm{MM}^{1}$, Ibrahim $\mathrm{M}^{2}$, Hiron $\mathrm{MM}^{1}$, Ahamad $\mathrm{M}^{3}$ \\ ${ }^{l}$ Department of Medcine, National Institute of Disease of the Chest and Hospital, Dhaka, Bangladesh \\ ${ }^{2}$ Department of Clinical Biochemistry, Lab Medicine Apollo Hospital, Dhaka, Bangladesh \\ ${ }^{3}$ Department of Respiratory medicine, Dhaka Medical College, Dhaka, Bangladesh
}

\section{Keywords:}

Adenosine deaminase; Pleural Effusion;

Tuberculosis

\begin{abstract}
Background: Tuberculosis is a leading cause of worldwide preventable morbidity and mortality from an infectious agent. A definite diagnosis of tubercular pleural effusion can be difficult to make because of low sensitivity and/or specificity of noninvasive traditional diagnostic tools. This study is done to find out a sensitive and specific marker for early diagnosis of tuberculous pleural effusion.
\end{abstract}

Materials and Methods: One hundred and three pleural effusion cases were enrolled in the study, out of which 62 were tuberculous pleural effusion cases and 49 were non-tuberculous cases.

Results: Considering $40 \mathrm{U} / \mathrm{L}$ as a cut off value for Adenosine deaminase assay level, the test result was positive in 58 out of 62 patients of tuberculosis indicating sensitivity of the test as $94 \%$; however, among 41 non-tuberculous patients, 5 presented ADA activity level more than 40U/L, which lowers the specificity of the test to $88 \%$. ADA levels were significantly higher in tuberculous than in non tuberculous cases (P value $<0.001)$.

Conclusion: It may be concluded that ADA levels are significantly high in patients with tuberculous pleural effusion compared to that in non tuberculous group. Sensitivity $(94 \%)$ and specificity $(88 \%)$ of the test in tuberculous pleural effusions are very high, when cut off value set at 40U/L. The result indicated that the analysis of ADA levels in pleural effusion constitute a very useful marker for the diagnosis of TPE which, in addition, can be made quickly in a non- invasive way.

\section{INTRODUCTION}

Tuberculosis (TB) is a disease of great antiquity. It is a leading causes of worldwide preventable morbidity and mortality from an infectious agent. Though pulmonary TB is the most common presentation of M. Tuberculosis infection, extrapulmonary TB also constitutes a frequent clinical problem,

\section{Correspondence:}

Dr. Shimul Kumar Bhowmik, MD

Registrar, Department of Medicine, National Institute of Disease of the Chest and Hospital, Dhaka, Bangladesh particularly due to advancing immunosuppressant in the present era of HIV infection. ${ }^{1}$ A pleural effusion occurs in approximately $5 \%$ of patients with TB. ${ }^{2}$

One study from Tanzania reported $38 \%$ of all tuberculosis cases exhibiting pleural involvement. ${ }^{3}$ In India extrapulmonary tuberculosis (EPTB) constitutes about 15$20 \%$ of all cases of TB in immunocompetent adult, among them tuberculous pleural effusion occurs in $20 \%$ cases. ${ }^{1}$ In Bangladesh, a study in a small scale was carried out in 2002, where the prevalence of EPTB was only $4.8 \%{ }^{4}$ So far no study regarding prevalence of tuberculous pleural effusion 
(TPE) was found to be done here.

A definite diagnosis of TPE can be difficult to make because of low sensitivity and/or specificity of noninvasive traditional diagnostic tools. ${ }^{5}$ Gold standard for diagnosis of pleural tuberculosis is the identification of M. tuberculosis in pleural fluid and tissue. ${ }^{6}$ But in clinical practice, this identification is problematic because of low identification rate of bacillus (less than $30 \%$ in the pleural fluid and approximately $50 \%$ in the pleural tissue) and slow growth of mycobacterium in culture (about 60 days). ${ }^{7}$ Direct analysis of pleural fluid for detection of acid-fast bacilli (AFB) by Ziehl-Neelsen (Z-N) or similar method is positive in less than $5 \%$ of cases and culture on Löwenstein-Jensen (L-J) medium does not surpass a $40 \%$ positivity rate. Moreover, direct examination of pleural fluid with Z-N staining requires bacilar concentration of $104 / \mathrm{ml}$ to be positive, but mycobacterial lode in pleural fluid is very low. ${ }^{8}$ In another study, smears of pleural fluid are positive in only $10 \%$ cases. ${ }^{9}$ Pleural fluid cultures are positive for mycobacteria in $<25 \%$ of cases. ${ }^{10}$ Moreover, culture is time consuming, requires 4-6 weeks to yield growth of M. Tuberculosis, even with radiometric mycobacterium culture system (BACTEC), which takes 18 days. Moreover cultures require a minimum of 10 to 100 viable bacilli. ${ }^{2}$

On the other hand, pleural biopsy demonstrates granulomatous pleuritis in $50-80 \%$ of patients with $\mathrm{TPE}^{11}$ and when a culture of biopsy specimen is combined with histological examination, the diagnosis can be established in approximately $90 \%$ of cases. ${ }^{6}$ In one study, a closed pleural biopsy specimen typically contained granulomas in only $60 \%$ of cases. ${ }^{12}$ Sometimes, in absence of granuloma, examination of biopsy specimen for AFB revealed organism in $10 \%$ of cases. ${ }^{13}$ Histopathology of pleural biopsy is superior to all the conventional test and is the specimen of choice for the diagnosis, ${ }^{14}$ but the risk of complications from thoracentesis, cost of patient care, the presence of a physician who is trained to perform the procedure and appropriate facilities for its performance, as well as a pathological anatomy laboratory and a experienced pathologist who can interpret the finding-all make the diagnostic procedure difficult. It does not always guarantee the collection of representative sample. Moreover, it is fully a blind invasive procedure.

Sputum specimens are often not evaluated because many of these patients are not able to produce sputum spontaneously. ${ }^{15}$ The sputum cultures are positive in $30-50 \%$ patients with both pulmonary and pleural tuberculosis. ${ }^{16}$

In this context, difficulty in diagnosing TPE led to a search for a simple, rapid, cost effective method that would optimize workup of those patients. Adenosine deaminase (ADA) assay may be very useful diagnostic approach for the diagnosis of TPE in combination with other diagnostic methods and clinical criteria.
Adenosine deaminase is an enzyme in the purine salvage pathway that catalyzes hydrolytic and irreversible deamination of deoxyadenosine and adenosine to deoxyinosine and inosine respectively with the release of ammonia. ${ }^{17}$ Although ubiquitous in distribution, enzyme activity is found in all cells with the highest activity in lymphocytes, predominantly active $\mathrm{T}$ lymphocytes and monocytes. ${ }^{18}$ ADA is involved in the proliferation and differentiation of lymphocytes, especially T lymphocytes. ${ }^{19}$ The increase in the ADA activity in patients with TB may indicate the cellular immune response and $\mathrm{T}$ lymphocyte activation in the disease. ${ }^{20} \mathrm{~T}$ lymphocytes have ADA level 10 to 12 times higher than B lymphocytes. ADA activity varies depending on the proliferative status and maturity of cells. ${ }^{21}$ The level of ADA is increased in TPE and this determination has acquired popularity as a diagnostic test in the high incidence area of TPE, because ADA measurement is less expensive, minimally invasive, rapid and readily accessible test.

\section{MATERIALS AND METHODS}

It was a cross sectional type of descriptive study. Samples were collected from inpatient department of National Institute of Diseases of The Chest and Hospital (NIDCH). The laboratory works were performed in the department of Microbiology \& Immunology, BSMMU and National Tuberculosis Reference Laboratory, NIDCH. The study was carried out during the period of January 2008 to December 2008. History relevant to pleural effusion was carefully taken and noted in predesigned.

\section{RESULTS}

One hundred and three pleural effusion cases were enrolled in the study. Out of the 103 cases, 62(60.1\%) were tuberculous pleural effusion cases and 49 (39.1\%) were non-tuberculous cases. Among the non-tuberculous cases, 30 cases were due to malignancy, 8 were due to pneumonia and rest 3 cases were due to nephrotic syndrome, congestive cardiac failure and rheumatoid arthritis each (Table 1).

According to the diagnostic criteria for patients of tuberculous pleural effusion, out of 62 patients diagnosed as having TPE, tuberculin test was positive in 42 cases (67.8\%), a pleural biopsy exhibited granulomatous inflammation in 27 cases $(43.5 \%)$ and chronic inflammatory lesion in 27 cases $(43.5 \%)$ for whom anti-TB drugs were

Table 1: Distribution of study population among pleural effusion cases

\begin{tabular}{cc}
\hline Diagnosis & No. of Cases (\%) \\
\hline Tuberculous pleural effusion & $62(60.1)$ \\
\hline Non-Tuberculous pleural effusion & $41(39.9)$ \\
Total & $103(100)$ \\
\hline Total & $\mathbf{1 0 6}$ \\
\hline
\end{tabular}


advised on the basis of clinical features, radiological evidence or empirically. Pleural fluid staining for AFB was positive in 6 patients $(9.6 \%)$, a pleural fluid culture for $\mathrm{M}$. tuberculosis was positive in 14 patients $(22.5 \%)$. Of the 20 patients who had productive cough, $2(10 \%)$ had positive sputum microscopy for AFB and 3(15\%) had positive sputum culture for M. tuberculosis. Out of 62 cases, only $5(8.06 \%)$ had evidence of pulmonary tuberculosis on chest $\mathrm{x}$-ray. Twenty two TPE cases were diagnosed by response to anti-tuberculous treatment (anti-TB) of which 13 cases were MT positive, and 9 cases had chronic inflammatory lesion on pleural tissue biopsy (Table 2).

Considering $40 \mathrm{U} / \mathrm{L}$ as a cut off value for ADA level, the test result was considered positive in 58 out of 62 patients of TB indicating sensitivity of the test as $94 \%$; however, among 41 non-tuberculous patients, 5 presented ADA activity level more than 40U/L, which lowers the specificity of the test to $88 \%$ (Table 3).

Adenosine deaminase activity showed the highest positive result in 58(93.5\%) TPE patients in comparison to all other tests (P value $<0.001$ ). Out of 62 cases, MT test was positive in 42 patients, histopathology of pleural tissue revealed granulomatous lesion in 27 patients and bacteriological methods confirmed TPE in 14 patients. Majority of TPE patients $48(77.41 \%$ ) were bacteriologically negative. Out of these 48 patients ADA result was positive in $46(95.83 \%)$ cases which was significantly higher $(\mathrm{P}$ value $<0.001)$ than biopsy positive cases $24(50.00 \%)$ and tuberculin test positive cases $30(62.5 \%)$. MT tests were done only in susceptive TPE cases which was a routine practice in NIDCH, Dhaka (Table 4).

Correlation and comparison of ADA result with bacteriological examination of pleural fluid, histopathology of pleural tissue and tuberculin test among the tuberculous pleural effusion cases was also done. (Table 5) Out of total 62 patients, fifty eight patients were positive for ADA. Among this 58 positive ADA patients, $6(10.34 \%)$ were positive for microscopy and $12(20.68 \%)$ were positive for culture; $27(46.55 \%)$ cases for pleural biopsy and $42(72.41 \%)$ cases for MT test were positive. Among the ADA negative cases $2(50 \%)$ were culture positive for M. tuberculosis. Other 2 $(50 \%)$ ADA negative cases were diagnosed by response to anti-tuberculous treatment that had chronic inflammatory lesion on histopathology of pleural biopsy.

ADA results in pleural fluid among the non- tuberculous pleural effusion cases were tabulated in table-VI. Among the 41 cases, $5(12.20 \%)$ were ADA positive of which $3(10 \%)$ cases were due to malignancy and one each was due to pneumonia and rheumatoid arthritis. Thirty six cases $(87.80 \%)$ were ADA negative.
Table 2: Distribution of tuberculous pleural effusion patients as per diagnostic criteria

\begin{tabular}{lcc}
\hline \multicolumn{1}{c}{ Diagnostic Criteria } & $\begin{array}{c}\text { Positive } \\
\text { Number }\end{array}$ & $\begin{array}{c}\text { Result } \\
\text { (\%) }\end{array}$ \\
\hline $\begin{array}{l}\text { Pleural Fluid } \\
\text { Culture for M.tuberculosis }\end{array}$ & 14 & $(22.5)$ \\
M/E for AFB & 6 & $(9.60)$ \\
Histopathology of Biopsized Pleural Tissue & & \\
$\quad$ Granulomatous inflammation & 27 & $(43.5)$ \\
$\quad$ Chronic inflammatory lesion & 27 & $(43.5)$ \\
Sputum (n=20)* & & \\
Culture for M.tuberculosis & 3 & $(15.0)$ \\
M/E for AFB & 2 & $(10.0)$ \\
Tuberculin test & 42 & $(67.7)$ \\
CXR & & \\
Evidence of pulmonary TB & 5 & $(8.06)$
\end{tabular}

Table 3: ADA level in pleural fluid of study groups

\begin{tabular}{lccc}
\hline \multicolumn{4}{c}{ Pleural effusion cases } \\
\hline $\begin{array}{l}\text { ADA Level in } \\
\text { pleual fluid }\end{array}$ & Tuberculous & Non-tuberculous & Total \\
\hline Positive & $58(93.5 \%)$ & $5(12.2 \%)$ & 63 \\
\hline Negative & $4(6.5 \%)$ & $36(87.8 \%)$ & 40 \\
\hline \multicolumn{1}{c}{ Total } & $\mathbf{6 2 ( 1 0 0 . 0 \% )}$ & $\mathbf{4 1 ( 1 0 0 . 0 \% )}$ & $\mathbf{1 0 3}$ \\
\hline
\end{tabular}

Table 4: Comparison of Bacteriological results in pleural fluid with histopathology of biopsized pleural tissue, MT test and ADA result in pleural fluid among tuberculous pleural effusion patients

\begin{tabular}{|c|c|c|c|}
\hline $\begin{array}{l}\text { Combined Result } \\
\text { of M/E for AFB \& } \\
\text { Culture for M.TwssB } \\
\text { in pleural fluid }\end{array}$ & $\begin{array}{l}\text { Positive } \\
\text { ADA in } \\
\text { pleural } \\
\text { fluid }\end{array}$ & $\begin{array}{c}\text { Positive } \\
\text { pleural } \\
\text { biopsy*for } \\
\text { granuloma }\end{array}$ & $\begin{array}{l}\text { Positive } \\
\text { MT test }\end{array}$ \\
\hline $\begin{array}{l}\text { Both } M / E \text { \&culture } \\
\text { positive } n=6\end{array}$ & $6(100 \%)$ & $2(33.33 \%)$ & $6(100 \%)$ \\
\hline $\begin{array}{l}\text { M/E negative \&culture } \\
\text { positive } n=8\end{array}$ & $6(75 \%)$ & $1(12.50 \%)$ & $6(75 \%)$ \\
\hline $\begin{array}{l}\text { M/E \&culture negative } \\
\mathrm{n}=48\end{array}$ & $46(95.83 \%)$ & $24(50.00 \%)$ & $30(62.5 \%)$ \\
\hline Total $\mathbf{N}=\mathbf{6 0}$ & & $27(43.54 \%)$ & $30(62.5 \%)$ \\
\hline
\end{tabular}

Adenosine deaminase activity levels obtained in pleural fluids of the studied groups were shown in figure I. The horizontal line shows the cut-off level. Mean ADA value in the group of TPE was $70.82 \pm 22.54 \mathrm{U} / \mathrm{L}$ versus 30.07 $\pm 22.93 \mathrm{U} / \mathrm{L}$ in non-tuberculous group and the difference is statistically highly significant $(\mathrm{p}<0.001)$. The ADA level of non-tuberculous group reached above the diagnostic cut-off $(40 \mathrm{U} / \mathrm{L})$ for TB in 5 of 41 cases (Two patients had bronchogenic carcinoma, one each had lymphoma, pneumonia and rheumatoid arthritis). Adenosine deaminase level of four TB patients were less than 40U/L. 
Table 5: Correlation of ADA results with bacteriological results in pleural fluid, histopathology of biopsized pleural tissue and MT test among tuberculous pleural effusion cases

\begin{tabular}{ccccc}
\hline $\begin{array}{c}\text { ADA in } \\
\text { pleural } \\
\text { fluid }\end{array}$ & $\begin{array}{c}\text { M/Efor } \\
\text { AFB } \\
\text { Positive }\end{array}$ & $\begin{array}{c}\text { Culture } \\
\text { for M. TB } \\
\text { Positive }\end{array}$ & $\begin{array}{c}\text { Biopsy for } \\
\text { Granu- } \\
\text { loma } \\
\text { Positive }\end{array}$ & $\begin{array}{c}\text { Tuberculin } \\
\text { Test Posi- } \\
\text { tive }\end{array}$ \\
\hline $\begin{array}{c}\text { ADA Posi- } \\
\text { tive } n=58\end{array}$ & $6(10.34)$ & $12(20.68)$ & $27(46.55)$ & $42(72.41)$ \\
$\begin{array}{c}\text { ADA } \\
\text { Negative } \\
\mathrm{n}=4\end{array}$ & $0(0.00)$ & $2(50.00)$ & $0(0.00)$ & $0(0.00)$ \\
\hline Total $\mathrm{n}=62$ & $6(9.62)$ & $\mathbf{1 4 ( 2 2 . 5 0 )}$ & $\mathbf{2 7 ( 4 3 . 5 4 )}$ & $\mathbf{4 2 ( 6 7 . 7 4 )}$ \\
\hline
\end{tabular}

Table 6: ADA results in pleural fluid among the nontuberculous pleural effusion cases

\begin{tabular}{|c|c|c|c|}
\hline \multicolumn{2}{|c|}{$\begin{array}{c}\text { NON-TUBERCULOUS } \\
\text { CASES }\end{array}$} & \multirow{2}{*}{$\begin{array}{c}\begin{array}{c}\text { ADA } \\
\text { POSITIVE }\end{array} \\
3(10.0)^{*}\end{array}$} & \multirow{2}{*}{$\begin{array}{c}\text { ADA } \\
\text { NEGATIVE } \\
27(90.0)\end{array}$} \\
\hline Malignant & $\mathrm{n}=30$ & & \\
\hline Pneumonia & $\mathrm{n}=8$ & $1(12.5)$ & $7(87.5)$ \\
\hline Rheumatoid Arthritis & $\mathrm{n}=1$ & $1(100)$ & $0(0.00)$ \\
\hline Nephrotic Syndrome & $\mathrm{n}=1$ & $0(0.00)$ & $1(100)$ \\
\hline Congestive Cardiac F & $\mathrm{e}=1$ & $0(0.00)$ & $1(100)$ \\
\hline Total & $n=41$ & $5(12.20)$ & $36(87.80)$ \\
\hline
\end{tabular}

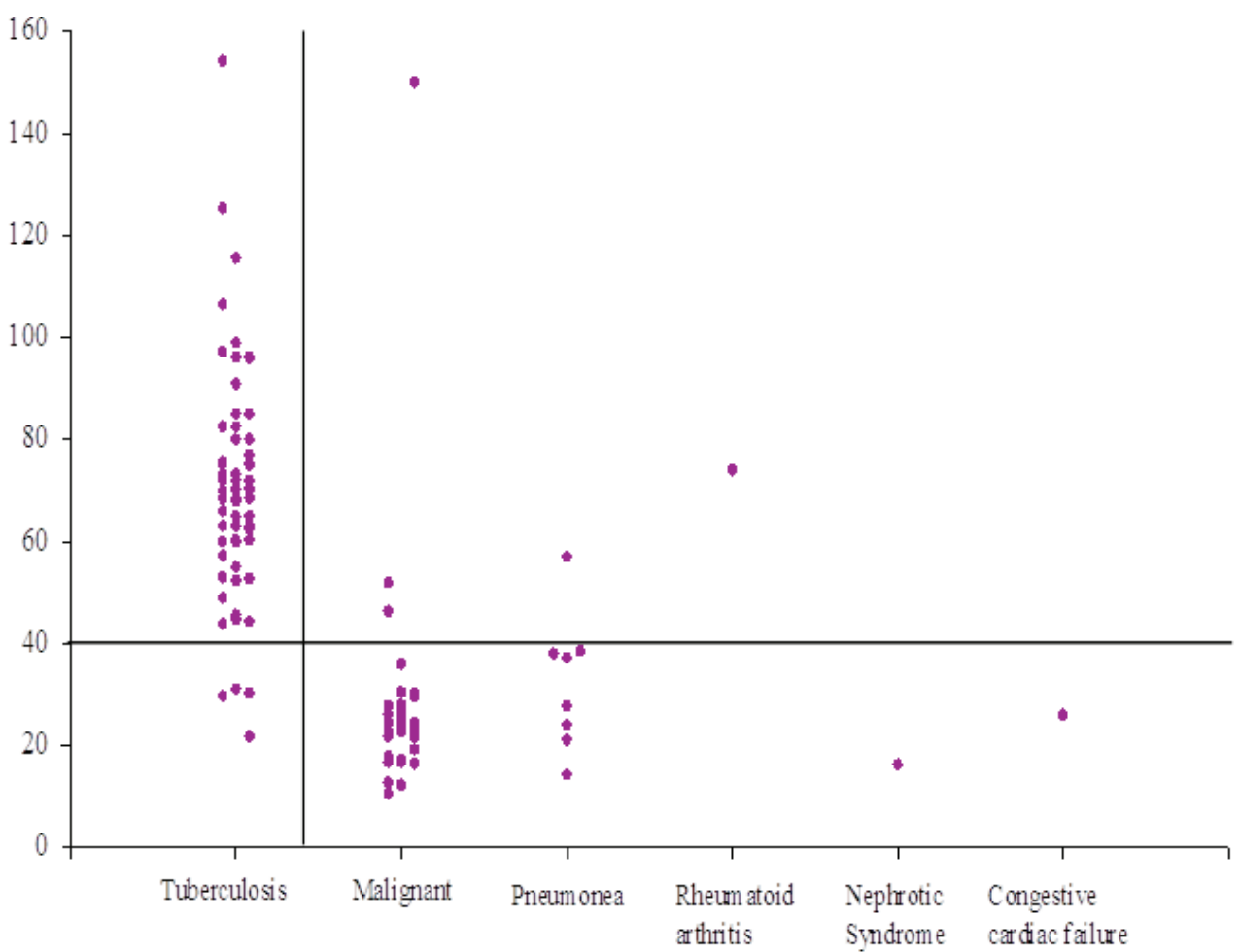

Figure I: Adenosine deaminase levels in pleural fluids of the studied groups.
Table 7 shows comparison of sensitivity, specificity, positive predictive value, negative predictive value and accuracy of ADA assay with every parameter studied for diagnosis of TPE. The specificity of ADA activity was $88 \%$, a value that is lower to that of other methods. However, in our patients, sensitivity of activity for diagnosis of tuberculous effusion was higher than that achieved with other methods. At lower cut-off value (like $33.56 \mathrm{U} / \mathrm{L}$ ), the sensitivity remains the same but the specificity falls to $78 \%$ which is also lower than that achieved with other methods. At higher cut-off value (like $45.2 \mathrm{U} / \mathrm{L}$ ), the sensitivity falls to $88 \%$ which is lower in comparison with values obtained through other methods but specificity rises to $87 \%$ which is near to the values obtained through other methods.

Table 8 shows the likelihood ratio for positive result is 7.66 which is much more away from 1.0 the baseline to determine the sensitivity. The likelihood ratio for negative result was 0.074 who indicate that a negative test has less chance to have disease which increases the specificity. At our cut-off value $(40 \mathrm{u} / \mathrm{L})$, the pre-test probability of ADA value for the diagnosis of TPE is $60 \%$ but the post-test probability at the same cut-off value $(40 \mathrm{u} / \mathrm{L})$ is $91 \%$ which indicate that the test is applicable for the diagnosis of TPE. 
Table 7: Comparison of Sensitivity, Specificity, Positive predictive value, Negative predictive value and Accuracy of ADA assay with every parameter studied for diagnosis of TPE

\begin{tabular}{|c|c|c|c|c|c|c|c|}
\hline & ADA level & Tuberculin test & Pleural biopsy & $\begin{array}{l}\text { Pleural fluid culture } \\
\text { for M.TB }\end{array}$ & Pleural fluid AFB & $\begin{array}{l}\text { Sputum culture } \\
\text { for M.TB }\end{array}$ & $\begin{array}{c}\text { Sputum } \\
\text { AFB }\end{array}$ \\
\hline Sensitivity & 0.94 & 0.68 & 0.44 & 0.23 & 0.10 & 0.15 & 0.10 \\
\hline Specificity & 0.88 & 0.93 & 1.00 & 1.00 & 1.00 & 1.00 & 1.00 \\
\hline PPV & 0.92 & 0.93 & 1.00 & 1.00 & 1.00 & 1.00 & 1.00 \\
\hline NPV & 0.90 & 0.66 & 0.54 & 0.46 & 0.42 & 0.69 & 0.68 \\
\hline Accuracy & 0.90 & 0.78 & 0.66 & 0.53 & 0.46 & 0.71 & 0.69 \\
\hline
\end{tabular}

Table 8: Determination of Likelihood Ratio, Pre-test probability \& post-test probability of ADA values in the diagnosis of Tuberculous Pleural Effusion

\begin{tabular}{lc}
\hline Likelihood Ratio for positive results & 7.66 \\
Likelihood Ratio for negative results & 0.074 \\
Pre-test probability & $60 \%$ \\
Post-test probability (LR+ve) & $91 \%$ \\
Post-test probability (LR-ve) & $10 \%$ \\
\hline
\end{tabular}

\section{DISCUSSION}

Tuberculous pleural effusion frequently represents a diagnostic problem even after extensive research. In the present study, the commonest cause of pleural effusion were found to be tuberculous $(60.2 \%)$ followed by malignancy (29.1\%) and pneumonia (7.7\%). These findings were similar to those observed in other studies. ${ }^{22}$ However this finding is in contrast with some other studies where they have found malignancy as the commonest cause of pleural effusion. ${ }^{23}$ Only a few cases of pleural effusion from congestive cardiac failure, rheumatoid arthritis and nephrotic syndrome were noted in the present study. Similar observations of rarity of pleural effusion by these diseases were also reported in Bangladesh. ${ }^{24}$

TPE represent an immunological reaction to relatively few acid-fast bacilli in the pleural space. Acid-fast bacilli are reported to be seen in less than $10 \%$ of pleural aspirates. ${ }^{24}$ $\mathrm{Z}-\mathrm{N}$ staining result of the pleural fluid were negative in all patient with TPE in two different study; in Brazil ${ }^{25}$ and in India ${ }^{26}$ In this study, Z-N stain of pleural fluid revealed positivity in $9.6 \%$ cases and pleural fluid culture yielded Mycobacterium tuberculosis in $22.5 \%$ cases. Culture requires a minimum of 10 to 100 viable bacilli and, therefore, is more sensitive than Z-N staining. ${ }^{26}$ Majority of series showed diagnostic yields of $<30 \%{ }^{27}$. Only one study by Sibley (1950) reported positive culture in $70 \%$ of the cases. Lower rate of culture positivity ranging $4-16 \%$ were also reported by other workers. ${ }^{28}$

Microscopic examination and culture of sputum seldom reveal AFB in patients with tuberculous effusion. ${ }^{29}$ The diagnostic value of culture for Mycobacterium tuberculosis in sputum of patients with pleural tuberculosis is generally considered low. ${ }^{26}$ Berger and Mejia reported that only 9\% $(2 / 22)$ of there patients, in whom no evidence of parenchymal lesion could be identified on the chest radiography, had a positive sputum culture. In another study, Arrington and colleague reported that none of 25 patients with tuberculous pleurisy had a positive sputum culture. In this study, sputum microscopy was diagnostic in 2 of $20(10 \%)$ and sputum culture in 3 of $20(15 \%)$ cases. Yew et al. reported $12.2 \%$ positive sputum microscopy for AFB in his study. Sputum culture for Mycobacterium tuberculosis was found to be positive in $16.7 \%$ cases in a study conducted in Brazil. ${ }^{30}$

Presence of granulomatous inflammation is frequently used as a diagnostic criteria for pleural tuberculosis and biopsy of the parietal pleura shows typical epithelioid granuloma in $50 \%$ to $80 \%$ of patients with TPE. ${ }^{31,32}$ In this study, it provided a positive diagnosis in $43.5 \%$ of TPE cases. Low rate of positivity in histological examination of pleural biopsy tissue was also reported by Desai $\mathrm{P}$ et al, who have found positive biopsy in only $38.4 \%$ cases. The low rate of positivity in the present study might be due to lack of repeat pleural biopsy in this series. Moreover, biopsy requires greater expertise and is subject to sampling error as epitheloid granulomas are not evenly distributed all over the pleural tissue. ${ }^{33}$

In this study, a ROC curve, a graphic approach, was employed which is preferable when there are many possible cut off value. More importantly, the use of a ROC curve in the present study permits a meaningful comparison of the sensitivity and specificity at different cut off values between our study and other studies.

In the present study, the mean ADA activity level in patients of TPE was $70.82 \pm 22.54 \mathrm{U} / \mathrm{L}$ while in the group of nontuberculous patients it was $30.07 \pm 22.93 \mathrm{U} / \mathrm{L}(\mathrm{P}<0.001)$. Valdes et al studied 129 cases of pleural effusion, out of which $81(62.8 \%)$ were of tuberculous etiology. The mean ADA level was significantly greater (112U/L) in tuberculous than non -tuberculous group. 
Of the 62 patients with TPE, 4 showed pleural ADA activities below the threshold value. Therefore an ADA measurement $<40 \mathrm{U} / \mathrm{L}$ does not preclude the possibility of TB. Two of the four patients with false negative result are associated with malignant condition, may be they had immunosuppression as evidenced by the negative tuberculin test. In another case, the patient had milliary tuberculosis on X-ray which occurs due to haematogenous dissemination. This may indicates an immunologic deficiency and deserves further study. False negative cases are frequently found in studies conducted for the diagnosis of TPE by ADA activity.

In this study, ADA showed $94 \%$ sensitivity and $88 \%$ specificity for the diagnosis of TPE that are compatible with other study results conducted by other research workers previously. This study showed likelihood ratio for positive result was 7.66 which move much away from 1.0 that indicates that more severe the disease, sensitivity of the test will be more. The likelihood ratio for negative results was 0.074 , again for away from 1.0, indicates that in patient who were negative for TPE has less chance to show positive results of ADA values that made ADA measurement a highly specific for TPE patients. So, the obtained results were applicable with high sensitivity and specificity for the TPE patients in practice. Moreover, high post-test probability $91 \%$ mean that the test result will determine the treatment plan more accurately. With higher ADA values $(>40 \mathrm{u} / \mathrm{L})$, the diagnosis will be more certain and treatment can be started. Post-test probability for negative results is $10 \%$ which indicates a negative ADA value can virtually exclude the diagnosis of TPE and antiTB can be deferred. This also strengthens the rationality to introduce ADA measurement as diagnostic tool in the management of pleural effusion. The value obtained by this study is sufficient enough to diagnose most of the cases of TPE within a short period (1-2 days). More over, it is least invasive, less costly, and comparably accurate and can be an easily available diagnostic procedure. Sample collection for ADA assay in also easy and can be done bedside and not necessitates any special arrangement. Chance of laboratory related errors is also less. Pleural fluid culture is also an important diagnostic test but at least 6 weeks are required to obtained results and creates a diagnostic delay. Pleural tissue biopsy and histopathology is an invasive, blind procedure and can be followed by several complications to patients and to results. Pleural fluid AFB staining has low sensitivity because presence of dead bacilli may cause false positive results and requires high bacillary load of 10,000 $/ \mathrm{ml}$ for positive result. So, considering all, ADA assay in pleural fluid can be considered as important investigation tool for the diagnosis of TPE.

\section{CONCLUSION}

In this study it has been clearly shown that ADA levels are significantly high in patients with tuberculous pleural effusion $(68.7 \pm 37.0 \mathrm{U} / \mathrm{L})$ compared to that $(28.6 \pm 8.3 \mathrm{U} / \mathrm{L})$ in non tuberculous group. Sensitivity (94\%) and specificity $(88 \%)$ of the test in TPE are very high, when cut off value set at $40 \mathrm{U} / \mathrm{L}$. The result indicated that the analysis of ADA levels in pleural effusion constitute a very useful marker for the diagnosis of TPE which, in addition, can be made quickly in a non- invasive way. However, ADA levels above the cut off level were found in $5(12.20 \%)$ of nontuberculous patients, one of them showed very high level and ADA levels remain below the cut off level in $4((6.5 \%)$ of TB patients. We may conclude that overall this test is very helpful test for the diagnosis of TPE.

\section{REFERENCES}

1. Sharma SK, Mohan A, Extrapulmonary tuberculosis. India J Med Res 2004;120:316-53.316-53.

2. Gopi A, Madhavan SM, Sharma SK and Sahn SA. Diagnosis and treatment of tuberculous pleural effusion in 2006. Chest 2007;131:880-9.

3. Mlika-Cabanne N, Brauner M, Muguisi F. Radiographic abnormalities in tuberculosis and risk of coex $\neg$ isting human immunodeficiency virus infection: results from Dares Salaam, Tanzania and scoring system. Am Rev Respir Crit Care Med 1995;152:786-93.

4. Talukder MAS and Zakaria MM. Prevalence of Extra-pulmonary tuberculosis in hospital patients. ASCON 2002;10:117.

5. Laniado-Laborin R. Adenosine deaminase in the diagnosis of tuberculous pleural effusion: Is it really an ideal test? A word of caution. Chest 2005;127:417-8.

6. Antonangelo L, Vargas FS, Almeida LP et al. Influence of storage time and temperature on pleural fluid adenosine deaminase determination. Respirology 2006;11:488-92.

7. Antonangelo L, Vargas FS, Seiscento $\mathrm{M}$ et al. Clinical and laboratory parameters in the differential diagnosis of pleural effusion secondary to tuberculosis or cancer. Clinics 2007;62:585-90.

8. Prabhudesai PP, Mahashur AA, Mehta N, Ajay R. Exudative pleural effusions in patients over forty years age- an analysis of seventy-six patients. J Postgrad Med 1993;39:190.

9. Khatami K. Pleural tuberculosis. Shiraz E-Medical Journal 2001;3:1 15 .

10. Laniado-Laborin R. Adenosine deaminase in the diagnosis of tuberculous pleural effusion: Is it really an ideal test? A word of caution. Chest 2005;127:417-8

11. Laniado-Laborin R. Adenosine deaminase in the diagnosis of tuberculous pleural effusion: Is it really an ideal test? A word of caution. Chest 2005;127:417-8.

12. Riantawan P, Chaowalit P, Wongsangiem M, Rojanaraweewong P. Diagnostic value of pleural fluid adenosine deaminase in tuberculous pleuritis with reference to HIV coinfection and a Bayesian analysis. Chest 1999;116:97-103.

13. Valdes L, Jose SE, Alvarez D et al. Diagnosis of tuberculous pleurisy using the biologic parameters adenosine deaminase, lysozyme and interferon gamma.Chest 1993;103:458-65.

14. Conde MB, Loivos CA, Rezende VM et al. Yield of sputum induction in the diagnosis of pleural tuberculosis. Am J Respir Crit Care Med $2003 ; 167: 723-5$.

15. Berger HW, Mejia E. Tuberculous pleurisy. Chest 1973;63:88-93.

16. Epstein DM, Kline LR, Albelda SM, Miller WT. Tuberculous pleural effusions. Chest 1987;91:106-9.

17. Ungerer JPJ, Oosthuizen Bissbort SH, Vermaak WJH. Serum adenosine deaminase: Isoenzymes and diagnostic application. Clin Chem 1992;38:1322-6. 
18. Laniado-Laborin R. Adenosine deaminase in the diagnosis of tuberculous pleural effusion: Is it really an ideal test? A word of caution. Chest 2005;127:417-8.

19. Canbolat O, Ulusdoyuran S, Ozgun G, Ceyhan I, Gumuslu F, Akbay A. The comparison of adenosine deaminase activity value with polymerase chain reaction results in patients with tuberculosis. J Clin Lab Anal1999;13:209-12.

20. Riquelme A, Calvo M, Salech F et al. Value of adenosine deaminase (ADA) in ascitic fluid for the diagnosis of tuberculous peritonitis. $\mathrm{J}$ Clin Gastroenterol 2006;40:705-10.

21. Carstens ME, Burgess LJ, Maritz FJ, Taljaard JJF. Isoenxymes of adenosine deaminase in pleural effusion: a diagnostic tool? Int $\mathrm{J}$ Tuberc Lung Dis 1998;2:831-5.

22. Reechaipichitkul W, Kawamatawong T, Teerajetgul Y, Patjanasoontorn B. Diagnostic role of pleural fluid adenosine deaminase in tuberculous pleural effusion. Southeast Asian J Trop Med Public health 2001;32:383-9.

23. Berger HW, Mejia E. Tuberculous pleurisy. Chest 1973;63:88-93.

24. Lima DM, Colares JKB, de Fonseca BAL. Combined used of the polymerase chain reaction and detection of adenosine deaminase activity on pleural fluid improves the rate of diagnosis of pleural tuberculosis. Chest 2003;24:909-14.
25. Prabhudesai PP, Mahashur AA, Mehta N, Ajay R. Exudative pleural effusions in patients over forty years age- an analysis of seventy-six patients. J Postgrad Med 1993;39:190.

26. Kaisemann MC, Kristski AL, Pereira MD, Trajman A. Pleural fluid adenosine deaminase detection for the diagnosis of pleural tuberculosis. J Bras Pneumol 2004;30:549-6.

27. Gopi A, Madhavan SM, Sharma SK, Sahn SA. Diagnosis and treatment of tuberculous pleural effusion in 2006. Chest 2007;131:880-9.

28. Yew Ws, Chan CY, Kwan SY, Cheung SW, GL French. Diagnosis of tuberculous pleural effusion by the detection of tuberculostearic acid in pleural aspirates. Chest 1991;100:1261-3.

29. Kumar D, Watson JM. Charlett A. Tuberculosis in England and Wales in 1993: results of a national sur $\neg$ vey. Thorax 1997;52:1060-7.

30. Seibert AF, Haynes J, Middleton R. Tuberculous pleural effusiontwenty year experience. Chest 1991;99:883-6.

31. Berger HW, Mejia E. Tuberculous pleurisy. Chest 1973;63:88-93.

32. Prabhudesai PP, Mahashur AA, Mehta N, Ajay R. Exudative pleural effusions in patients over forty years age- an analysis of seventy-six patients. J Postgrad Med 1993;39:190.

33. Valdes L, Alvarez D, Jose ES et al. Value of adenosine deaminase in the diagnosis of tuberculous pleural effusions in young patients in a region of high prevalence of tuberculosis. Thorax 1995;50:600-3. 